\title{
Requirements of orbital phase continuity revisited: A FMO approach
}

\author{
Yuji Naruse*,1,2 \\ ${ }^{1}$ Department of Chemistry and Biomolecular Science and ${ }^{2}$ Department of Materials Chemistry and Processing, Gifu Univer- \\ sity, 1-1 Yanagido, Gifu 501-1193 JAPAN \\ KEYWORDS (Word Style "BG_Keywords"). If you are submitting your paper to a journal that requires keywords, provide \\ significant keywords to aid the reader in literature retrieval.
}

\begin{abstract}
Cyclic orbital interaction, in which a series of orbitals interact with each other so as to make a monocyclic system, affords stabilization if the requirements of orbital phase continuity are satisfied. Initially, these requirements were derived from the consideration of a three-body system. Here I propose that these requirements can be easily derived by considering FMO theory.
\end{abstract}

Recently, we reported that the phase of the orbitals ${ }^{1}$, which originates from the wave character of electrons in molecules, plays an essential role in determining reactivity and selectivity. Especially, cyclic orbital interaction ${ }^{2}$, in which a series of orbitals interact in a monocyclic manner, determines the regioselectivity in deprotonation of an indole sidechain ${ }^{3,4}$, diastereoselectivity in pericyclic reactions ${ }^{5}$ including the electrocyclic ringopening reaction of 3 -substituted cyclobutenes ${ }^{6,7}$, cheletropic reactions ${ }^{8}$ and the retro-Nazarov reaction. ${ }^{9,10}$ Recently, we reported that diastereoselectivity in the uncatalyzed Mukaiyama aldol reaction ${ }^{11}$ and the electrophilic addition of $\alpha$-substituted ethylenes ${ }^{12}$ are under the control of cyclic orbital interaction.

In these reactions, the phase-continuous cyclic orbital interaction stabilizes the TS, so that the reactions prefer to proceed in the direction that gives greater stabilization. To determine whether the cyclic orbital interaction is phase-continuous, the series of orbital interactions should satisfy the requirements of orbital phase continuity: (i) donating orbitals are out-of-phase; (ii) a donating orbital and an accepting orbital are in-phase; and (iii) accepting orbitals are in-phase (Figure 1). When the number of corresponding orbitals in the interaction is more than 3, two additional requirements should also be satisfied: (iv) interaction among the orbitals is monocyclic, and (v) the series of orbital interactions should be divided into two, a donor part and an acceptor part, and not into four, six or more. Note that the overlap between the orbitals is less than $1 ;|\mathrm{S}|<1$, cyclic orbital interaction with fewer orbitals is often preferred.

\section{(i) Donating orbitals are out-of-phase.}

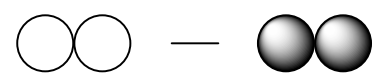

(ii) A donating orbital and an accepting orbital are inphase.

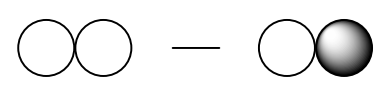

(iii) Accepting orbitals are in-phase.

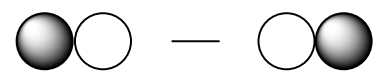

(iv) For interaction among more than three systems, the cyclic orbital interaction is monocyclic, i.e., the bonds interact with adjacent bonds but only little or negligibly with those in a remote position.

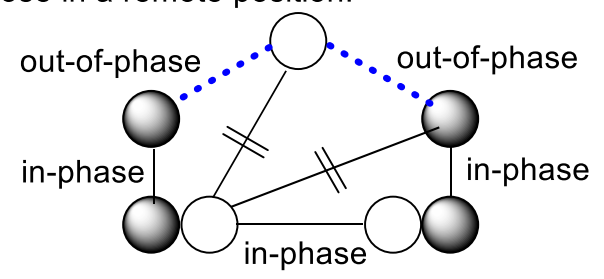

(v) The cyclic orbital interaction must be divided into only two parts, the donor $\boldsymbol{D}$ and acceptor $\boldsymbol{A}$ parts, and not into four, six, or so on.
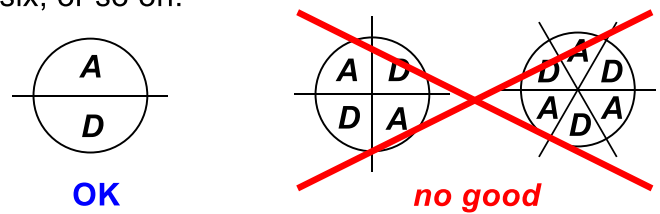

Figure 1. The requirements of orbital phase continuity for cyclic orbital interaction.

Inagaki derived these requirements by considering the perturbation of interaction in a three-body system which consists of one occupied orbital in Body A and two vacant orbitals in Bodies B and C, respectively (eq. 1, Figure 2, shown for delocalization of the $\beta$-spin electron only). 
$H_{O O^{\prime}}(3 r d)=$
$\sum_{K, L(\neq K)} \frac{\left(H_{O K}-S_{O K} H_{O O}\right)\left(H_{L O}-S_{L O} H_{O O}\right)\left(H_{K L}-S_{K L} H_{O O}\right)}{\left(H_{O O}-H_{K K}\right)\left(H_{O O}-H_{L L}\right)}$

where $\boldsymbol{O}$ is the electronic state for the ground configuration and $\boldsymbol{O}^{\prime}$ is that with some perturbation from the ground state. The electronic states for $\boldsymbol{K}$ and $\boldsymbol{L}$ are those with delocalization. Electrons are delocalized among the electronic states of $\boldsymbol{O}, \boldsymbol{K}$ and $\boldsymbol{L}$.

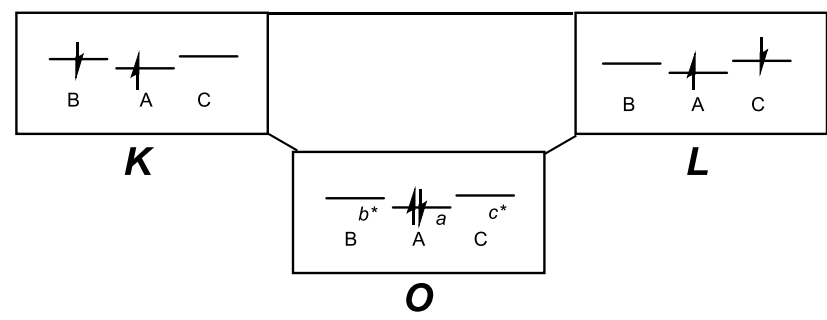

Figure 2. Delocalization among the electronic configurations of $\boldsymbol{O}$, $\boldsymbol{K}$ and $\boldsymbol{L}$, in which the bodies consist of a donor A and two acceptors $\mathrm{B}$ and $\mathrm{C}$.

He also concluded that consideration of a combination of two donors and one acceptor leads to the same conclusion.

Although the requirements of orbital phase continuity themselves are quite easy to understand, this approach is still somewhat difficult to comprehend since it requires the use of complicated equations with perturbation theory. Thus, its applications are still limited. Here I show that the FMO theory can also easily explain the requirements for orbital phase continuity.

First, we consider the case in which a series of orbital interactions is monocyclic (Requirement (iv)) and the system can be divided into one donor and one acceptor (Requirement (v)). Under these conditions, the donor and the acceptor always interact at two points (Figure 3 ).

\section{FMO interaction}

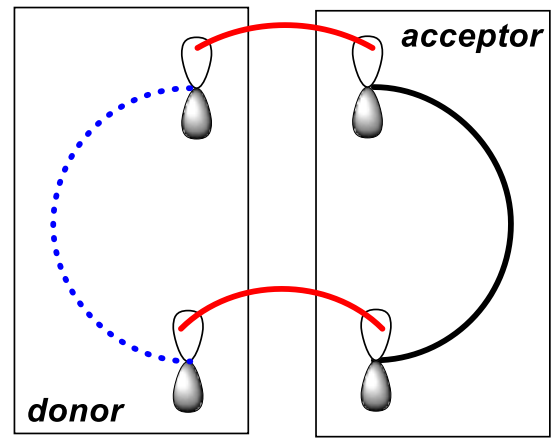

Figure 3. FMO interaction in cyclic orbital interaction.

The FMO interaction, a stabilizing interaction, is always a donor-acceptor interaction, so that it should be an in-phase combination of the orbitals, which corresponds to Requirement (ii).

When two orbitals interact, a pair of new orbitals are created: one with in-phase combination, which is lower in energy, and another that is out-of-phase and higher in energy (Figure 4). For example, when two hydrogen atoms form a hydrogen molecule, a pair of new molecular orbitals, which consist of two 1s orbitals, are created. In a hydrogen molecule, the molecular orbital and the bond orbital are the same. The orbital lower in energy is the in-phase combination of two hydrogen 1s orbitals. The other orbital, which is higher in energy, is the out-of-phase combination. Two electrons occupy the $\sigma$-orbital, the molecular orbital that is lower in energy, to make a bond between two hydrogen atoms, stabilizing the system.

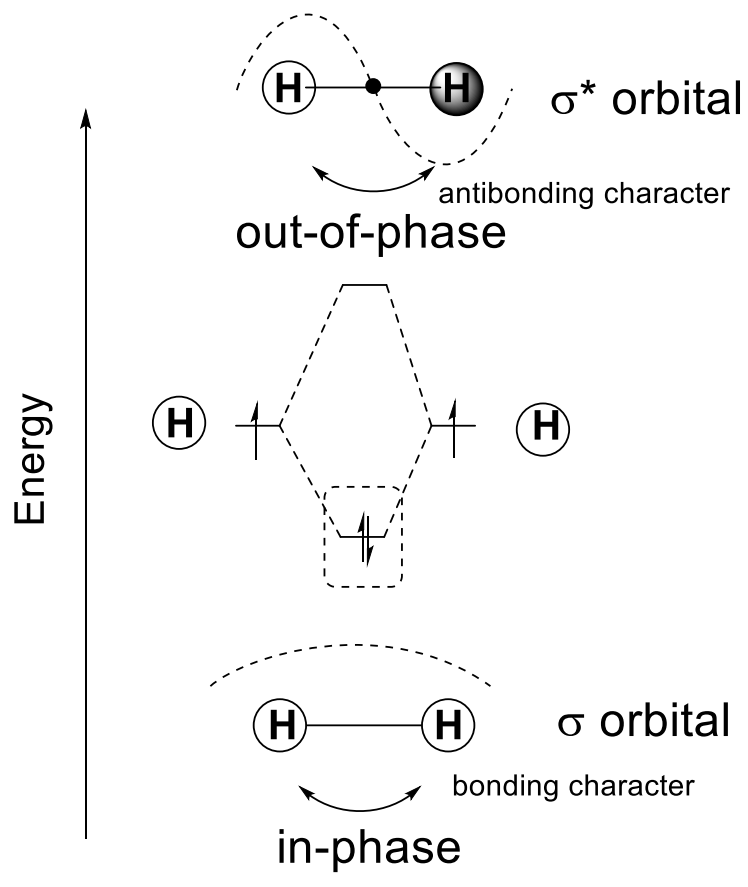

Figure 4. Molecular orbital of $\mathrm{H}_{2}$ from the hydrogen atomic orbital.

Now, the donor part consists of donating orbitals only. Thus, the orbital combination with the most electron-donating character, i.e., with the highest orbital energy (=HOMO), is an all outof-phase combination, where the number of phase alternations is $N_{\mathrm{D}}$. This corresponds to Requirement (i). On the other hand, the acceptor consists of accepting orbitals only. The orbital combination with the most electron-withdrawing character, i.e., with the lowest orbital energy (=LUMO), is an all in-phase combination, where the number of phase alternations is $N_{\mathrm{A}}$. This is Requirement (iii).

For the phase-continuous cyclic orbital interaction, the total number of phase alternations in the orbital combination $N_{\mathrm{D}}+N_{\mathrm{A}}$ is an even number. In contrast, that in the orbital combination is odd when the interaction is phase-discontinuous. From the perspective of a wave, the condition in which the system satisfies all these requirements of orbital phase continuity is when a standing wave is formed. Thus, with the topological requirement of a monocyclic ring, the total number $N_{\mathrm{D}}+N_{\mathrm{A}}$ should be even, or, in the case of an odd number, the wave cannot resonate and the interaction should be negated. This is the most important point of the theory.

This discussion shows that the requirements of orbital phase continuity can be easily derived with a consideration of FMO interaction. It heavily depends on the phase of the orbital, which is based on the wave character of the electron. In chemistry, the view that an electron acts as a particle is still predominant, especially in the field of organic chemistry. Forty years have passed since Fukui's FMO theory and the Woodward-Hoffmann rules were honored by the Nobel Prize. These theories more strongly emphasize the wave character of electrons in molecules. I believe that further consideration of the wave character will help us to guide new perspectives and insights to design new molecules and reactions. 
AUTHOR INFORMATION

Corresponding Author

*e-mail: naruse@gifu-u.ac.jp.

\section{REFERENCES}

1. Inagaki, S. in Orbitals in Chemistry Springer, Berlin-Heidelburg, 2009.

2. Inagaki, S. "An Orbital Phase Theory" Top. Curr. Chem. 2009, 289, 83-127.

3. Naruse, Y.; Ito, Y.; Inagaki, S. "Highly Regioselective Functionalization of 2,3-Dialkyl Substituents on Indoles" J. Org. Chem. 1991, 56, 2256-2258.

4. Naruse, Y.; Kido, K.; Inagaki, S. "Ethylation of the Indole Dianion by Alkyl Methyl Ethers" J. Org. Chem. 1996, 60, 8334-8335.

5. Review: Naruse, Y.; Inagaki, S. "Participation of Geminal Bonds in Organic Reactions" Chem. Lett. 2007, 820-825.

6. Ikeda, H.; Kato, T.; Inagaki, S. Geminal Bond Participation in the Electrocyclic Reactions: Torquoselectivities of Silyl and Trimethylsilyl Derivatives of Cyclobutene, Iminocyclobutene and Cyclobutenone" Chem. Lett. 2001, 270-271.
7. Yasui, M.; Naruse, Y.; Inagaki, S. “An Orbital Phase Theory for the Torquoselectivity of the Ring-Opening Reactions of 3-Substituted Cyclobutenes: Geminal Bond Participation” J. Org. Chem. 2004, 69, $7246-7249$

8. Naruse, Y.; Hayashi, Y.; Inagaki, S. "Geminal bond participation and torquoselectivity in cheletropic reactions" Tetrahedron Lett. 2003, $44,8509-8512$.

9. Naruse, Y.; Ichihashi, Y.; Shimizu, T.; Inagaki, S. "Predominance of Participation of the Geminal over Vicinal Bonds. Torquoselectivity of Retro-Nazarov Reactions” Org. Lett. 2012, 14, 3728-3731.

10. Naruse, Y.; Tokunaga, M. "Geminal bond participation is essential for the contradictory torquoselectivities in retro-Nazarov reactions" Tetrahedron Lett. 2015, 56, 3813-3815.

11. Naruse, Y.; Fukasawa, S.; Ota, S.; Deki, A.; Inagaki, S. "Geminal Bond Participation in the Uncatalyzed Mukaiyama Aldol Reactions" Tetrahedron Lett. 2007, 48, 817-820.

12. Naruse, Y.; Hasegawa, Y.; Ikemoto, K. "Orbital theory for diastereoselectivity in electrophilic addition" Tetrahedron Lett. 2016, 57, 2029-2033. 